\title{
Nonlinear Optical Properties of Liquid Crystals Probed by Z-scan Technique
}

\author{
S.L. Gómez, F.L.S. Cuppo, and A.M. Figueiredo Neto \\ Complex Fluid Group, Institute of Physics \\ University of São Paulo, SP, Brazil
}

Received on 22 May, 2003

\begin{abstract}
We present a review of experimental data on the nonlinear optical properties of thermotropic and lyotropic liquid crystals probed by the Z-scan technique. Depending on the time scale, different processes originate a nonlinear optical response. Particularly, at nanosecond range, the physical processes associated with the nonlinear response in thermotropics are not completely understood. In lyotropics, the nonlinear response at ms time scale is from thermal origin and depends on the particular mesophase, relative concentration of the components, and temperature.
\end{abstract}

\section{Introduction}

Liquid crystals (LC) are known to exhibit large optical nonlinearities which have been the subject of considerable study in recent years, from both experimental and theoretical points of view. So far, almost all the research has been focused on thermotropic liquid crystals (TLC) [1,2]. Since LC molecules typically have delocalized $\pi$-electrons, they are believed to be potential sources of fast and large nonlinearities. Due to bulk anisotropy of nematic LC, nonlinear optical parameters (e.g., nonlinear refraction index $n_{2}$ and nonlinear optical absorption $\beta$ ) are expected to depend on the geometrical configuration between the light polarizing direction $(\mathbf{E})$ and the nematic director $\widehat{n}$.

However, only few works have been devoted to lyotropic liquid crystals (LLC). LLC are mixtures of amphiphilic molecules and a solvent which, at given temperature and relative concentration conditions, self-assembly in anisotropic aggregates. These aggregates can have different forms and, if their shape anisotropy is small, they are called micelles [3]. In nematic LLC made by at least two types of amphiphilic molecules and a solvent, micelles present an orthorhombic symmetry and most of the particularities of lyotropic mesophases are related to the properties of these building blocks [4]. A characteristic of the nematic LLC, which is not observed in thermotropics, is the existence of a biaxial $N_{b x}$ mesophase, between a calamitic $N_{c}$ and a discotic $N_{d}$ nematic mesophases [5].

Nonlinear optical phenomena can be due to electronic and nonelectronic processes [6]. The former refers to those radiative interactions between the active electron and the optical electric field. Usually, they are very fast, of the order of $p s$, and spatially localized. Examples are second- and thirdharmonic generation. Nonelectronic processes are nonradiative interactions such as temperature-, density-, and or- der parameter-changes, cis-trans isomerism, phase transition and director axis-reorientation. These mechanisms usually do not involve generation of new frequency radiation. Several techniques have been developed to measure the nonlinear optical refractive index $n_{2}$ (nonlinear interferometry, degenerate four-wave mixing and ellipse rotation, among others). These techniques are sensitive but usually require relative complex experimental apparatus. The single-beam Z-scan technique, an application developed by the CREOL group [7], is simple (from the experimental point of view) and sensitive in measuring both the nonlinear refraction and nonlinear absorption. It is in a powerful and straightforward technique to study nonlinear properties of materials in different time scales.

The aim of this work is to present an overview of the nonlinear optical research in TLC and LLC by using the Zscan technique. The Z-scan technique will be described and the origin of the nonlinear optical properties at different time scales will be discussed.

\section{The Z-scan technique}

In this technique, a polarized Gaussian laser beam, propagating in the $z$-direction, is focused to a narrow waist. The sample is moved along the $z$-direction and the transmitted intensity is measured through a finite aperture in the far field as a function of the sample position $z$, measured with respect to the focal plane. As the sample moves through the beam focus (at $z=0$ ), self-focusing or -defocusing modifies the wave front phase, thereby modifying the detected beam intensity. A schema of the setup of the Z-scan technique is depicted in Fig. 1. 


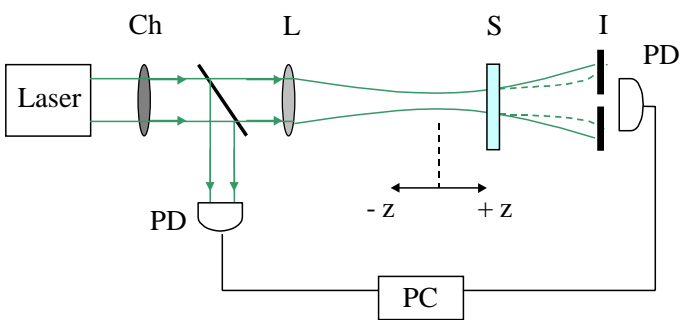

Figure 1. Schema of the experimental setup of the Z-scan technique: chopper (Ch); lens (L); sample (S); iris (I) and photo detector (PD).

To figure out how the Z-scan transmittance as a function of $z$ is related to the nonlinear refraction of the sample, let us assume a medium with a negative nonlinear refraction index and a thickness smaller than the diffraction length of the focused beam. This can be considered as a thin lens of variable focal length. Beginning far from the focus $(z<0)$, the beam irradiance is low and nonlinear refraction is negligible. In this condition, the measured transmittance remains constant (i.e., $z$-independent). As the sample approaches the beam focus, irradiance increases, leading to self-lensing in the sample. A negative self-lens before the focal plane will tend to collimate the beam on the aperture in the far field, increasing the transmittance measured at the iris position. After the focal plane, the same self-defocusing increases the beam divergence, leading to a widening of the beam at the iris and thus reducing the measured transmittance. Far from focus $(z>0)$, again the nonlinear refraction is low resulting in a transmittance $z$-independent. A pre-focal transmittance maximum (peak), followed by a post-focal transmittance minimum (valley) is a Z-scan signature of a negative nonlinearity. An inverse Z-scan curve (i.e., a valley followed by a peak) characterize a positive nonlinearity. Fig. 2 depicts these two situations.

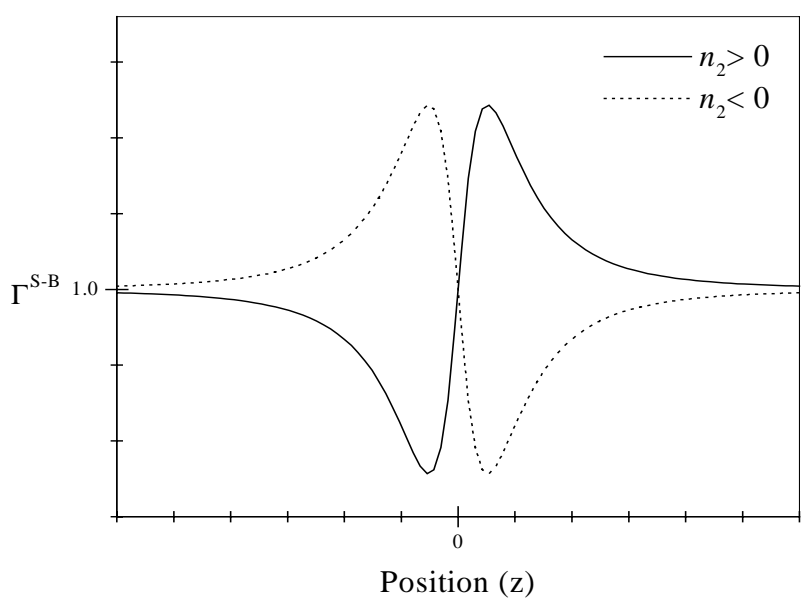

Figure 2. Z-scan theoretical curves of the transmittance as a function of $z$, obtained with Eq. 5

\section{Electronic nonlinear optical process}

Let us consider a medium subjected to an (optical) electric field $\mathbf{E}$. The induced polarization $(\mathbf{P})$ can be written as:

$$
\mathbf{P}=\varepsilon_{\mathbf{o}}\left(\chi^{(\mathbf{1})} \mathbf{E}+\overleftrightarrow{\chi}^{(2)}: \mathbf{E E}+\overleftrightarrow{\chi}^{(3)}: \mathbf{E E E}+\ldots\right)
$$

where $\chi^{(i)}$ is the electrical susceptibility of order $i$ and $\varepsilon_{o}$ is the permittivity of the free space [8]. Considering centrosymmetric media and keeping up to third-order terms in Eq. 1, from the Maxwell's equations one obtains the refractive index $n$ and the optical absorption $\alpha$ as functions of the intensity $I$ of the incident laser beam [1]:

$$
\begin{aligned}
n & =n_{o}+\frac{n_{2}}{2} E^{2}=n_{0}+\gamma I, \\
\alpha & =\alpha_{o}+\beta I,
\end{aligned}
$$

where $n_{o}$ and $\alpha_{o}$ are the linear refractive index and linear optical absorption respectively, $n_{2}$ is a coefficient named the nonlinear refractive index and $\beta$ is the nonlinear optical $a b$ sorption coefficient. The coefficients $\gamma$ and $\beta$ are related to the real and imaginary parts, respectively, of third-order electrical susceptibility $\chi^{(3)}$ by:

$$
\begin{aligned}
\gamma & =\frac{1}{2 n_{o}^{2} \varepsilon_{o} c} \operatorname{Re}\left(\chi^{(3)}\right) \\
\beta & =\frac{\omega}{n_{o}^{2} \varepsilon_{o} c^{2}} \operatorname{Im}\left(\chi^{(3)}\right) .
\end{aligned}
$$

A comprehensive discussion about units used in nonlinear optics can be found in ref. [9].

For a sample with negligible nonlinear absorption, the on-axis normalized transmittance $\Gamma^{S-B}$ as a function of $z$ is given, approximately, by [7]:

$$
\Gamma^{S-B}(z)=\frac{S(z)}{S(z \rightarrow \infty)} \simeq 1+\Delta \Phi_{o} \frac{4 x}{\left(1+x^{2}\right)\left(9+x^{2}\right)},
$$

where $S(t)$ is the photodetector signal at time $t, \Delta \Phi_{o}$ is the on-axis nonlinear phase shift at focus, $x=\frac{z}{z_{o}}$ and $z_{o}$ is the Rayleigh range of the Gaussian beam. The nonlinear phase shift is given by:

$$
\Delta \Phi_{o}=\frac{2 \pi d}{\lambda} \gamma I_{o}
$$

where $\lambda$ is the light wavelength, $I_{o}$ is the on-axis intensity at focus, $d$ is the sample thickness and $c$ is the speed of light at vacuum.

Note that equation 2 is "local", i.e., the refractive index at a given point on the material depends only on the light intensity at the same point.

The electronic nonlinear optical phenomena usually have response time of the order of femto- to picoseconds. 
Values of the nonlinear refraction index and nonlinear absorption, obtained in the picoseconds time scale in 5CB (4cyano-4'-n-pentylbiphenyl) in the nematic phase, at different wavelengths by Palffy-Muhoray et al. [10, 11, 12], are shown in Table 1.

The macroscopic susceptibility of third order $\chi^{(3)}$ is linearly related to the microscopic second-order hyperpolarizability $\gamma$ [13]. Thermotropic liquid crystals are usually organic compounds and these compounds interact weakly through van der Waals forces. Carbons can form two types of bonds: covalent $\sigma$-bonds, spatially localized and $\pi$-bonds, regions of delocalized electronic charge. The electronic density of $\pi$-bonds is much more mobile than that of the $\sigma$-bond. So, the nonlinear optical properties mainly depend on the change of polarizability of the electrons in the $\pi$-bonding orbitals. Hermann et al.[14] shown that the nonlinear optical properties of organic compounds with electrons $\pi$ in their structures are bigger than those of the saturated compounds with the same number of carbon atoms. They shown also that $\gamma \propto n_{\sigma}$, where $n_{\sigma}$ is the number of electrons $\sigma$ and is nonlinear in the number of $n_{\pi}$. The model of free electrons for the $\pi$-bonding orbitals [15] predicts that, in the case of a conjugated chain, $\gamma \propto n_{\pi}^{5}$. In the case of $5 \mathrm{CB}$, the nonlinear optical response is due mainly to the $\pi$-electrons of the benzene rings.

Table 2 shows values of $n_{2}$ and $\beta$, obtained studying the nonlinear optical response in time scale of nanoseconds, of the 5CB, 8CB, E7 and CB15 thermotropic liquid crystals in the nematic phase [10]. For some of these TLC, e.g., $5 \mathrm{CB}$, a strong nonlinear refraction and absorption have been observed but the mechanism that originate them are not yet well understood. The nonlinear refraction $n_{2}$ was shown to be fluence-dependent [18] and the nonlinear absorption $\beta$ is intensity-dependent, suggesting that different processes are responsible for them [12]. Moreover, $n_{2}$ does not depend on
$I$, indicating that it originates from a third-order process. On the other hand, $\beta$ depends linearly on $I$ indicating, a fifthorder process . The nonlinear absorption shows a fast response time (less than $10 \mathrm{~ns}$ and larger than $30 \mathrm{ps}$ ) suggesting a two-photon excited-state absorption. This conclusion is supported in the particular case of the 5CB since its linear absorption is very small. On the other hand, the magnitude and time-dependence of $n_{2}$ is consistent with those originated from a thermal mechanism. The only shortcoming, a value of $n_{2}$ independent on $T$ on approaching the transition temperature $T_{c}$ to the isotropic phase, could be explained by a slowing down of the orientational relaxation for $T \rightarrow T_{c}$ [17]. At these short time scales, many puzzles remain, like the unexpected large value of $\beta$ at nanosecond time scale, 10 - to $10^{2}$ times larger than that in the picosecond time scale [16]. To account for this result, it was suggested the possibility of formation of $\mu \mathrm{m}$-sized bubbles and microscopic carbonization in the sample during the laser exposure. Other important features that influence the magnitude and sign of the nonlinear refraction index are the beam waist and the thickness of the sample (ref. [16] and references therein). In the case of the isotropic phase of the 5CB it was observed that increasing the beam waist from $7 \mu \mathrm{m}$ to $35-50 \mu \mathrm{m}$, $n_{2}$ changes from negative to positive. This difference is explained by the dependence of the value of transient thermaldensity nonlinearity with negative sign on beam-waist diameter. The buildup time $t_{a c}$ of the thermal-density nonlinearity $\left(\Delta n_{\rho}=(\partial n / \partial \rho) \Delta \rho\right)$ is given by $t_{a c}=r_{o} / V_{s}$, where $r_{o}$ is the beam radius and $V_{s}$ is the velocity of the sound ( $\sim 1.5 \times 10^{3} \mathrm{~m} / \mathrm{s}$ in liquid crystals). For a $7 \mu \mathrm{m}$ diameter beam waist, $t_{a c}$ is less than the pulse duration. The negative nonlinear refraction is due to density changes of the medium, which overcomes the positive-sign of the transient orientational nonlinearity.

Table 1: Nonlinear refraction and nonlinear absorption of $5 \mathrm{CB}$, at $24^{\circ} \mathrm{C}$, with light pulses width of $33 p s$, at different wavelengths. HGA and HTA mean homogeneous planar alignment and homeotropic alignment, respectively. ${ }^{(1)}$ ref. [10]; ${ }^{(2)}$ ref. [12].

\begin{tabular}{|c|c|c|c|c|c|c|}
\hline$\lambda(n m)$ & $n_{2 \|}\left(10^{-11}\right.$ esu $)$ & $\beta_{\|}(\mathrm{cm} / G W)$ & \multicolumn{2}{|c|}{$n_{2 \perp}\left(10^{-11}\right.$ esu $)$} & \multicolumn{2}{|c|}{$\beta_{\perp}(\mathrm{cm} / G W)$} \\
\hline "532(1) & $+1.36^{(\mathrm{HGA})}$ & $2.27^{\text {(HGA) }}$ & $+0.6^{(\mathrm{HGA})}$ & 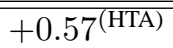 & $\overline{0.8^{\text {(HGA) }}}$ & 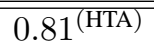 \\
\hline $600^{(2)}$ & $+1.9^{(\mathrm{HGA})}$ & $2.67^{(\mathrm{HGA})}$ & \multicolumn{2}{|c|}{ NA } & \multicolumn{2}{|c|}{ NA } \\
\hline $1064^{(2)}$ & $+0.55^{(\mathrm{HGA})}$ & 0 & \multicolumn{2}{|c|}{$+0.55^{(\mathrm{HGA})}$} & \multicolumn{2}{|c|}{0} \\
\hline
\end{tabular}

Table 2: Values of nonlinear refraction index and absorption of selected TLC at $532 \mathrm{~nm}$ and $7 \mathrm{~ns}$ light pulse width (ref. [11]). Columns 2-5 are for nematic phases and 6-7 for LCs in the isotropic phase.

\begin{tabular}{|l|c|c|c|c|c|c|}
\hline TLC & $n_{2 \|}\left(10^{-11}\right.$ esu $)$ & $\beta_{\|}(\mathrm{cm} / G W)$ & $n_{2 \perp}\left(10^{-11}\right.$ esu $)$ & $\beta_{\perp}(\mathrm{cm} / \mathrm{GW})$ & $n_{2}$ (iso) & $\beta$ (iso) \\
\hline \hline 5CB & -54 & 265 & 8.3 & 36 & -24 & 114 \\
\hline 8CB & -26 & 246 & 3.7 & 20 & - & - \\
\hline E7 & -38 & 284 & 3.3 & 40 & - & - \\
\hline CB15 & - & - & - & - & 7.4 & 38 \\
\hline
\end{tabular}




\section{Nonelectronic nonlinear optical processes}

Although light absorption is a local process, the diffusion of heat gives rise to a nonlinear optical phenomenon which are nonlocal in nature. This indirect nonlinear phenomenon is common to all absorbing materials but in liquid crystals it has an additional characteristics due to their peculiar orientational ordering.

Considering a cw Gaussian beam, the energy absorbed is immediately converted into heat. The continuous heat transferred to the sample gives rise to the formation of a radially Gaussian-shaped temperature profile. The hotter region is located in the center of the beam ( $r=0$, being $r$ the radial distance from the beam axis). The index of refraction $n$ of the medium, as a function of $r$ and the time $t$, can be written as:

$$
n(r, t)=n_{o}+\frac{\partial n}{\partial T} \Delta T(r, t)
$$

where $\Delta T$ is the temperature increase in the sample and $\partial n / \partial T$ is the thermooptic coefficient.

Depending on the time scale of the experiment, one should take into account the thermodiffusion process or Soret effect $[19,20]$. The Soret effect is characterized by a concentration gradient induced by a temperature gradient present in a medium. Due to the fact that in a mixture (e.g., the case of the LLC) the index of refraction depends on the concentration of solute, the gradient of matter generates a "lens of matter", in analogy with the thermal lens [21]. Usually, the diffusion of heat and mass have characteristic times of $\mathrm{ms}$ and $\mathrm{s}$, respectively [22].

The effects of local heating (due to light absorption) on the propagation of a Gaussian beam were studied by Gordon et al. [23]. This study is the foundation of the Thermal Lens model (TLM). Carter et al. [24] showed that the normalized transmittance in a Z-scan experiment, considering a radial diffusion of heat and the parabolic approximation of the temperature field, can be written as:

$$
\begin{aligned}
\Gamma^{T-L}(z) & =\frac{S(z, t=0)-S(z, t=\infty)}{S(z, t=\infty)} \\
& =\theta \frac{2 x}{1+x^{2}}+\theta^{2} \frac{1}{1+x^{2}},
\end{aligned}
$$

where $\theta$ is the on-axis nonlinear phase shift (i.e., the difference between its value in the center and in the outer edge of the beam) written as:

$$
\theta=\frac{\alpha_{o} P}{\lambda \kappa}\left(-\frac{\partial n}{\partial T}\right)
$$

where $\alpha_{o}$ is the linear absorption, $\lambda$ and $P$ the wavelength and the power of the incident beam, respectively, and $\kappa$ is the thermal conductivity. The time-evolution of the thermal lens signal is given by:

$$
S(t, z)=S(t=0, z)\left\{1+\frac{\theta}{1+t_{c} / 2 t} \frac{2 x}{1+x^{2}}+\left[\frac{\theta}{1+t_{c} / 2 t}\right]^{2} \times \frac{1}{1+x^{2}}\right\}^{-1}
$$

where $t_{c}(z)$ is a characteristic relaxation time at position $z$, given by:

$$
t_{c}(z)=\frac{\omega^{2}(z)}{4 D},
$$

where $\omega(z)=\left[\omega_{o}^{2}\left(1+x^{2}\right)\right]^{1 / 2}$ is the radius of the beam at position $z$ and $D$ is the thermal diffusivity of the medium.

\subsection{Thermotropic Liquid Crystals}

In TLC, measurements made at ms time scale in 5CB, in a geometry where reorientation is not expected, show that nonlinear optical response originates from laser heating due to linear absorption (nonlinear optical absorption is not observed) [11]. The increase in the samples's temperature decreases the degree of orientational order and density, resulting that in most liquid crystals $n_{2 \|}<0$ and $n_{2 \perp}>0$ (the symbols $॥$ and $\perp$ represent the director $\widehat{n}$ parallel and perpendicular to $\mathbf{E}$, respectively). Typical values for 5CB (10 ms laser pulse, $\left.T=24^{\circ} \mathrm{C}, \lambda=514 \mathrm{~nm}\right)$ are $n_{2 \|}=-10 \times 10^{-4}$ esu and $n_{2 \perp}=2.0 \times 10^{-4}$ esu. However, for ZLI-1538 and T15 thermotropics, both nonlinear refractive indices are negative $\mathrm{T} 15\left[\mathrm{~T}=180^{\circ} \mathrm{C}\right]: n_{2 \|}=$ $-0.68 \times 10^{-4}$ esu, $n_{2 \perp}=-0.092 \times 10^{-4}$ esu; ZLI1538 $\left[T=70^{\circ} \mathrm{C}\right]: n_{2 \|}=-0.43 \times 10^{-4} e s u, n_{2 \perp}=-0.13 \times$ $10^{-4}$ esu. It was suggested that conjugation of bonds and conformational changes play some role in these processes.

\subsection{General features of the nonlinear optical response of $L L C$}

At ms time scale, the nonlinear optical response of LLC has a thermal origin. It depends on the absorption properties of the sample: type of amphiphilic molecules, relative concentration, phase type, temperature and eventual ferrofluid doping.

Figure 3 shows the typical response of a lyotropic sample subjected to a square laser light pulse, at three different 
positions: $z<0, z=0$ and $z>0$. This behavior characterizes a negative nonlinear medium. Figure 4 shows a typical $\mathrm{Z}$-scan curve (normalized transmittance as a function of $z$ ) of a LLC and a fitting function using Eq. 6.

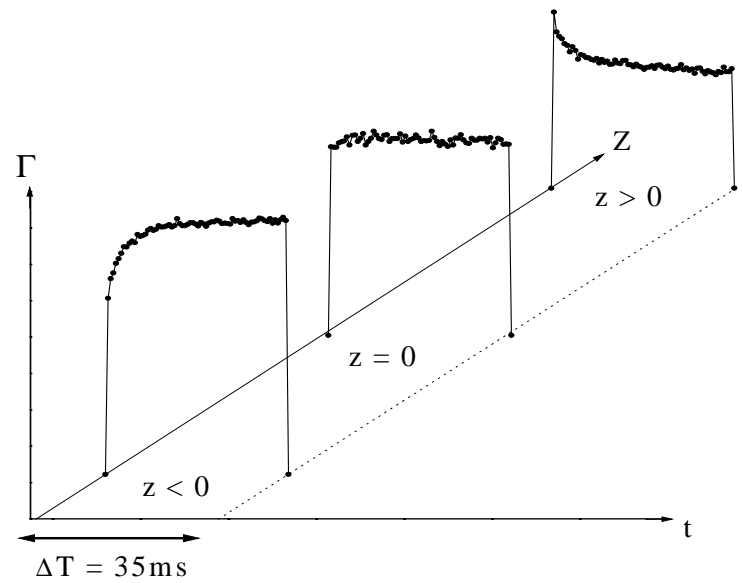

Figure 3. Typical oscilloscope's records of the transmittance, as a function of time, of a lyotropic liquid crystal sample, with $n_{2}<0$, when illuminated with a square pulse of $\sim 35 \mathrm{~ms}$, in three $z$ positions. Sample in the calamitic nematic phase, with a small ferrofluid doping, at room temperature $\left(T=23^{\circ} \mathrm{C}\right)$, a polarization of the beam $\mathbf{E} \| \widehat{n}$.

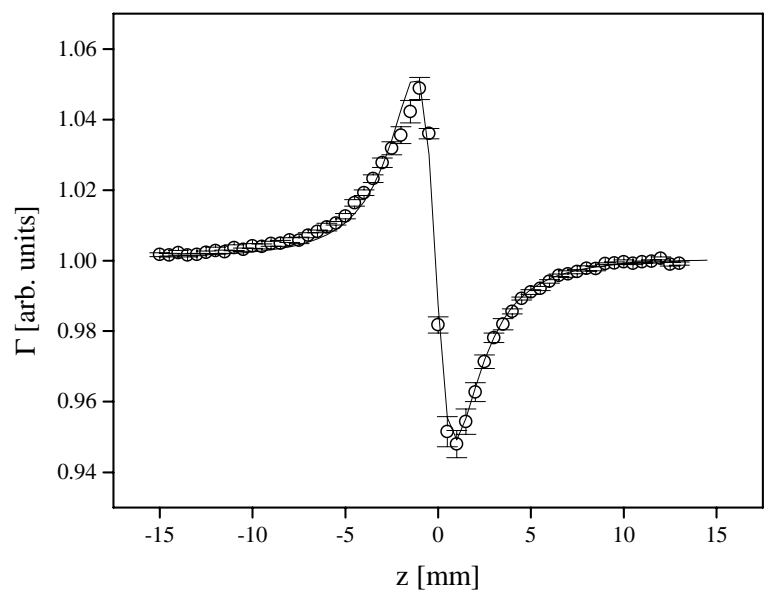

Figure 4. Typical Z-scan curve of a LLC normalized according to Sheik-Bahae model. Solid line corresponds to a fitting according to Eq. 6). Sample in the calamitic nematic phase, with a small ferrofluid doping, at room temperature $\left(T=23^{\circ} \mathrm{C}\right)$, polarization of the beam $\mathbf{E} \| \widehat{\mathbf{n}}$.

Lyotropic liquid crystals in the calamitic nematic $\left(N_{c}\right)$ and isotropic phases were shown to present nonlinear refractive index $n_{2} \sim-10^{-6}$ esu [25, 26], which is $10^{2}$ smaller than that measured in $5 \mathrm{CB}$ in the same time scale [10]. At the $N_{c}$ phase, $n_{2}<0$ for both configurations between the nematic director and the electric field polarization of the beam. On the other hand, the values of the thermooptic coefficient and the thermal conductivity are usually $\frac{d n}{d T} \sim 10^{-5 \circ} \mathrm{C}^{-1}$ and $\kappa \sim 10^{-1} \mathrm{~W} / \mathrm{m}^{\circ} \mathrm{C}$, respectively. The anisotropy observed in the values of these parameters (II and $\perp)$ are related to microscopic properties and local ordering of the amphiphilic aggregates [27].

\subsection{Effect of the ferrofluid doping}

Brochard and de Gennes [28] suggested that liquid crystals could be oriented by much weaker magnetic fields (about $10^{3}$ times smaller) if small magnetic grains were incorporated into the liquid crystalline matrix. Surfacted ferrofluids [29] are stable colloidal suspensions of small surfactant coated magnetic grains, typical dimensions of $10 \mathrm{~nm}$, dispersed in a polar or nonpolar liquid carrier. It has been observed that ferrofluid-doped LLC samples are stable over long periods of time. This doping, in small quantities (concentration of ferrofluid $c \lesssim 10^{13}$ grains $/ \mathrm{cm}^{3}$ ), does not modify some optical properties as well as the phase transition temperatures of LLC [30]. Besides that, the optical absorption of the ferrofluid-doped LLC significantly increases and, consequently, its nonlinear optical response.

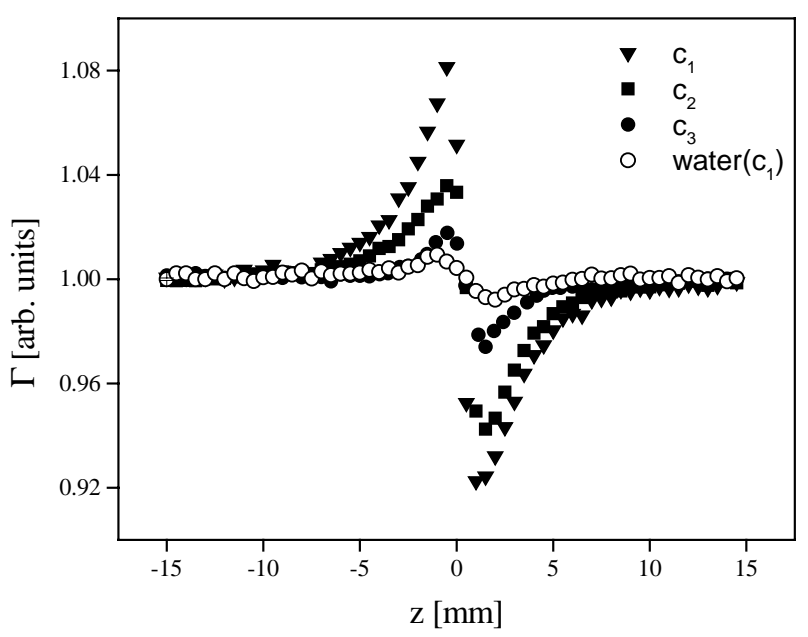

Figure 5. Experimental Z-scan curves of LLCs in the isotropic phase (KL[27.041], DeOH [6.409],water [66.550]) at room temperature $\left(\sim 25^{\circ} \mathrm{C}\right)$, with different doping concentrations $\left(c_{i=1,2,3}\right)$ of ferrofluid. In the figure $c_{1}>c_{2}>c_{3}$.

Figure 5 shows Z-scan curves of a lyotropic mixture in the isotropic phase (composition in weight\%: potassium laurate (KL) [27.041], 1-decanol (DeOH) [6.409] and water [66.550]), for different doping concentrations of ferrofluid. At the same Fig. 5 it is shown the Z-scan curve for a pure ferrofluid solution, with a concentration of grains of the same order than the maximum value used in the doped lyotropic sample. As can be seen from Fig. 5, the strength of the thermal lens, i.e. $n_{2}$, increases with the ferrofluid doping. It was also observed that the characteristic relaxation time $t_{c}$ and the thermooptic coefficient $d n / d T$ diminish with increasing the ferrofluid doping, while the value of the thermal conductivity $\kappa$ increases with the ferrofluid doping [31]. 


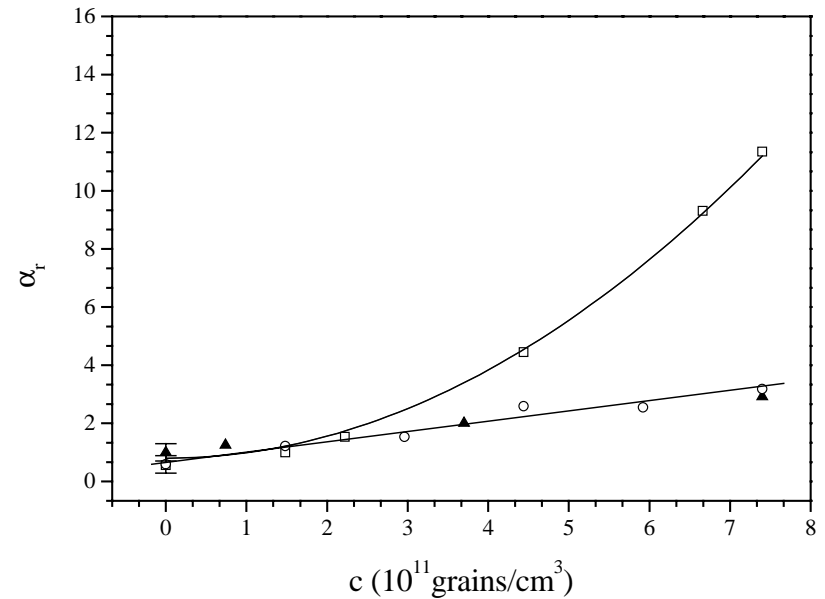

Figure 6. Linear optical absorption as a function of the ferrofluid concentration: $(\boldsymbol{\Lambda})$ pure ferrofluid, i.e., magnetic grains in water; (O) KL/water, at a concentration of KL smaller than the critical micellar concentration; ( $\square$ ) KL/1-DeOH/water LLC in the isotropic phase. From ref. [32].

The origin of the enhancement of the optical absorption was also investigated [32]. Fig. 6 shows the linear optical absorption of three ferrofluid-doped systems as a function of the ferrofluid grains concentration: a binary mixture of $\mathrm{KL}$ and water, at a concentration of KL smaller than the cmc [33]; pure ferrofluid (i.e., magnetic grains in water); and a lyotropic mixture (KL/DeOH/water) in the isotropic phase. It was observed that in the case of the pure ferrofluid and the binary of KL and water, the optical absorption increases linearly with the ferrofluid doping concentration $c$ as predicted by the Beer-Lambert law [34]. On the other hand, the behavior of the optical absorption of the ternary lyotropic mixture deviates from the usual Beer-Lambert law. This result clearly shows that the presence of micelles in the system is fundamental to determine the behavior of the linear absorbance as a function of $c$. A possible mechanism that could account for this behavior could be the hyperthermia [35], which is an indirect heating of the micelles via the magnetic grains. Micelles, having more degrees of freedom (fluctuation of the form, diffusion of molecules at the bilayer, etc.) seem to absorb more than the monodisperse amphiphilic molecules solution. This process could also be one of the responsible for the increase in the nonlinear response of LLC doped with ferrofluids.

\subsection{Influence of the amphiphilic aggregates}

The origin of the microscopic mechanisms responsible for the nonlinear optical response of LLC are not yet well known. As mentioned above, the existence of micelles modifies the linear absorbance behavior of the ferrofluid-doped mixture and could also account for the nonlinear optical response of the lyotropic liquid crystals. As a general remark one can assert that the presence of micelles modify the thermooptic properties of a medium. Z-scan measurements done by Cuppo et al. in a binary mixture of KL and water [36], show that $n_{2} \sim$ cte. at KL concentrations
$\left(c_{K L}\right)$ below the $c m c$ and increases linearly with $c_{K L}$ for $c_{K L}>\mathrm{cmc}$, reaching a saturation value for $c_{L K} \sim 100 \mathrm{cmc}$ (see Fig. 9 in ref. [36]). The values of $n_{2}$ for this binary mixture are two orders of magnitude smaller than those of the ternary $\mathrm{KL} / \mathrm{DeOH} /$ water mixture in the isotropic and nematic phases. Comparing this result with the known dependence of the different physical parameters of amphiphilic solutions at concentrations around the $\mathrm{cmc}$ [37], the absolute values of the thermooptic coefficient $\left(\left|\frac{\partial n}{\partial T}\right|\right)$ and of the nonlinear refractive index $\left(\left|n_{2}\right|\right)$ present, qualitatively, the behaviors of the high frequency electric conductivity $\left(\sigma_{H F}\right)$ and the inverse of the equivalent electric conductivity $\left(\sigma_{E Q}\right)$. The counterions mobility in the double layer around the micelles seems to be strongly related to the nonlinear response of the medium to an electric field.

\subsection{Nonlinear birefringence at the $N_{c}-I$ and $N_{c}-N_{b x}$ phase transitions}

de Gennes showed that the linear birefringence is an adequate (scalar) order parameter for liquid crystals [38]. Usually, temperature-driven transitions are determined measuring the linear birefringence by polarized optical microscopy. The dielectric tensor $\overleftrightarrow{\epsilon}$ can be written as function of the linear birefringences $\Delta n_{L}$ and $\delta n_{L}$ [39]. The measurement of the linear birefringences around the transition temperature $\left(T_{c}\right)$, give informations about the collective behavior of the basic units (molecules or micelles) of the system. Galerne $e t$ al. [39] show that the $N_{c}-I$ (here, $I$ states for the isotropic phase) phase transition is weakly first-order and the linear birefringence has a discontinuity at $T_{c I}$ (here, $T_{c I}$ states for the $N_{c}-I$ transition temperature). On the other hand, the $N_{c}-N_{b x}$ phase transition is second-order and mean-field. Saupe et al. [5] observed a deviation from the mean-field behavior at the $N_{c}-N_{b x}$ transition in a range of temperatures $\frac{\Delta T_{c}}{T_{c}} \sim 10^{-4}$ from $T_{c}$, obtaining $\beta=0,38(3)$ for the value of the critical exponent of the order parameter.

Cuppo et al. [41] investigated the behavior of the nonlinear refractive indices $\left(n_{2}\right)$ and nonlinear birefringence $\left(\Delta n_{2}\right)$ in the vicinity of the $N_{c}-I$ and $N_{c}-N_{b x}$ phase transitions in a LLC. Their results at the $N_{c}-I$ transition indicate that the sign of the thermooptic coefficient remains the same up to $0.1^{\circ} \mathrm{C}$ from $T_{c I}$. The values of $n_{2}$ in both configurations of the laser electric field $\mathbf{E}$ with respect to the magnetic field $\mathbf{H}$ used to orient the nematic: parallel and perpendicular, are negative. These results are rather different from those obtained by Pereira et al. [42], in a similar lyotropic mixture, using the Thermal Lens technique. A possible origin for this discrepancy could be related to the orientation of the $N_{c}$ phase sample during the measurement. In the case of the work of Cuppo et al., the magnetic orienting field is present during the all the experiment, differently from the one of Pereira et al.. In the work of Cuppo et al., the nonlinear optical birefringence in the $N_{c}$ phase, $\left(\Delta n_{2}=n_{2 \|}-n_{2 \perp}\right)$ shows a small discontinuity at $\mathrm{T}_{c I}$, consistent with a weak first-order phase transition. In the $N_{c}-N_{b x}$ transition it was observed a singular-type behavior 
of $n_{2}(/ /$ and $\perp)$ at the transition temperature $T_{c N}$ [32]. The nonlinear birefringence was shown to present a divergence behavior of the type $\Delta n_{2} \sim\left|T-T_{c N}\right|^{-\beta}$ with $\beta \sim 0.5$, in both sides ( $N_{c}$ and $N_{b x}$ phase domains) of the phase transition temperature. An inversion of the sign of $n_{2||}$ at the $N_{b x}$ phase was observed in a particular mixture composition of the same mixture. The same behavior was observed in a lyotropic mixture of KL/decylammonium chloride/water in the $N_{b x}$ phase [43]. The origin of this inversion is not yet understood.

\section{Concluding remarks}

Although the research of liquid crystals nonlinear optical properties is in the beginning, many of the basic properties involved in these processes have already been discovered. Employing the Z-scan technique it was observed that, in $m s$ time scales, the origin of the nonlinear optical response is thermal and depends on the basic parameters that rule out the mesophases like temperature and relative composition, among others. Two remarkable features are the strong dependence of the nonlinear optical response on the existence of micelles and the nonlinear enhancement with the ferrofluid doping in micellar mesophases. The deviation from the phenomenological Beer-Lambert law denotes the existence of an interaction between micelles and magnetic grains whose nature is not yet well understood. Finally, the observation of a convergent thermal lens in a biaxial nematic phase could indicate the influence of the geometry of the biaxial aggregates and of the phase itself, opening an interesting subject of research both theoretically and experimentally.

\section{Acknowledgments}

We thank FAPESP and CNPq/PRONEX for the financial support of this research.

\section{References}

[1] I.C. Khoo and S.T. Wu, Optics and Nonlinear Optics of Liquid Crystals (World Scientific, Singapore 1993).

[2] F. Simoni, Nonlinear Optical Properties of Liquid Crystals and Polymer Dispersed Liquid Crystals (World Scientific, Singapore 1997).

[3] Phase Transitions in Complex Fluids, ed. P. Tolédano and A.M. Figueiredo Neto (World Scientific, Singapore 1998).

[4] Y. Galerne, A.M. Figueiredo Neto, and L. Liébert, J. Chem. Phys. 87, 1851 (1987).

[5] L.J. Yu and A. Saupe, Phys. Rev. Lett. 45, 1000 (1980).

[6] I.C. Khoo, Liquid Crystals: Physical Properties and Nonlinear Optical Phenomena, (Wiley, New York 1995).

[7] M. Sheik-Bahae, A.A. Said, T.H. Wei, D.J. Hagan, and E.W. Van Stryland, IEEE J. Quantum Electron. 26, 760 (1990).

[8] Y.R. Shen, The Principles of Nonlinear Optics (Wiley, New York 1984)
[9] R.W. Boyd, Nonlinear Optics (Academic Press 1992).

[10] P. Palffy-Muhoray, H.J. Yuan, L. Li, M.A. Lee, J.R. DeSalvo, T.H. Wei, M. Sheik-Bahae, D.J. Hagan, and E.W. Van Stryland, Mol. Cryst. Liq. Cryst. 207, 291 (1991).

[11] L. Li, H.J. Yuan, G. Hu, and P. Palffy-Muhoray, Liquid Crystals 16, 703 (1994).

[12] P. Palffy-Muhoray, T.H. Wei, and W. Zhao, Mol. Cryst. Liq. Cryst. 251, 19 (1994).

[13] Ch. Bosshard, in Nonlinear Optical Effects and Materials, ed. by P. Günter (Springer, Berlin 2000).

[14] J.P. Hermann, D. Ricard, and J. Ducuing, Appl. Phys. Lett. 23, 178 (1973).

[15] P.N. Prasad and D.J. Williams, Introduction to Nonlinear Optical Effects in Molecules and Polymers, (Wiley-Interscience, New York 1991).

[16] S.G. Lukishova, J. Nonlin. Opt. Phys. Mat. 9, 365 (2000).

[17] C.W. Greeff, J. Lu, and M.A. Lee, Liquid Crystals, 15, 75 (1993).

[18] Fluence of an electromagnetic field is defined as the timeintegral of the intensity $I$ of the electromagnetic field.

[19] Ch. Soret, Arch. Sci. Phy. Nat., 2, 48 (1879).

[20] S.R. De Groot, L'Effet Soret, diffusion thermique dans les phases condensées, Noord-Hollandsche Uitgevers Maatschappij, Amsterdam, (1945).

[21] S. Alves, A. Bourdon, and A.M. Figueiredo Neto, J. Opt. Soc. Am. B 20, 713 (2003).

[22] J. Georges and T. Paris, Anal. Chim. Acta 386, 287 (1999).

[23] J.P. Gordon, R.C.C. Leite, R.S. Moore, S.P.S. Porto, and J.R. Whinnery, J. Appl. Phys. 36, 3 (1965).

[24] C.A. Carter and J.M. Harris, Appl. Opt. 23, 476 (1984).

[25] S.L. Gómez, PhD Thesis, University of São Paulo, Brazil 2000.

[26] S.L. Gómez, F.L.S. Cuppo, A.M. Figueiredo Neto, T. Kosa, M. Muramatsu, and R.J. Horowicz, Phys. Rev. E 59, 3059 (1999).

[27] S.L. Gómez and A.M. Figueiredo Neto, Phys. Rev. E 62, 675 (2000).

[28] F. Brochard and P.G.D Gennes, J. Phys-Paris 31, 691 (1970).

[29] R.E. Rosensweig, Ferrohydrodynamics (Cambridge Univ. Press, Cambridge, 1985).

[30] A.M. Figueiredo Neto, Y. Galerne, A.M. Levelut, and L. Liébert in Physics of Complex and Supermolecular Fluids, EXXON Monograph Series, edited. by S.A. Safran and N.A. Clark (Wiley, New York, 1987), p. 347.

[31] F.L.S. Cuppo, PhD Thesis, University of São Paulo 2002.

[32] F.L.S. Cuppo, S. Gomez and A. M. Figueiredo Neto, submitted.

[33] The $c m c$ (critical micelar concentration) is defined as the minimum concentration of amphiphilic molecules in a solvent above which amphiphilic molecules self assembly in nanoscopic aggregates.

[34] J.R. Lakowicz, Principles of Fluorescence Spectroscopy (Plenum Press, New York, 1983). 
[35] A. Jordan, P. Wust, R. Scholz, B. Tesche, H. Fähling, T. Mitrovics, T. Vogl, J. Cervós-Navarro, and R. Felix, Int. J. Hyperthermia 12, 705 (1993).

[36] F.L.S. Cuppo and A.M. Figueiredo Neto, Langmuir 18, 9647 (2002).

[37] W.C. Preston, J. Phys. Colloid Chem. 52, 84 (1948).

[38] P.G. de Gennes, The Physics of Liquid Crystals (Clarendon Press, Oxford, 1975).

[39] Y. Galerne and J.P. Marcerou, Phys. Rev. Lett. 51, 2109 (1983).
[40] F.L.S. Cuppo, A.M. Figueiredo Neto, S.L. Gómez, and P. Palffy-Muhoray, J. Opt. Soc. Am. B 19, 1342 (2002).

[41] F.L.S. Cuppo, S.L. Gómez, and A.M. Figueiredo Neto, Phy. Rev. E 67, 051711 (2003).

[42] J.R.D. Pereira, A.J. Palangana, A.M. Mansanares, E.C. da Silva, A.C. Bento, and M.L. Baesso, Phys. Rev. E 61, 5410 (2000).

[43] I.H. Bechtold, S.L. Gómez, J.J. Bonvent, Th. Rasing, and E.A. Oliveira, submitted. 